\title{
Iron deficiency anemia in children with congenital heart disease and the usefulness of erythrocyte indexes as a screening tool.
}

\author{
Truong Quang Binh ${ }^{1}$, Nguyen Thi Bang Suong ${ }^{2}$, Nguyen Hoang Dinh ${ }^{1}$, Vo Tuan Anh ${ }^{1}$, Vu Tri Thanh ${ }^{1}$, Le Minh Khoi ${ }^{{ }^{*}}$ \\ ${ }^{1}$ Cardiovascular Center, University Medical Center, University of Medicine and Pharmacy at Ho Chi Minh City, Vietnam \\ ${ }^{2}$ Department of Laboratory, University Medical Center, University of Medicine and Pharmacy at Ho Chi Minh City, Vietnam
}

\begin{abstract}
Introduction: The iron deficiency anemia (IDA) is the most common micronutrient malnutrition in the world. This condition has been shown to increase morbidity in adults and to exert negative impact on the psycho-motor development in children. The IDA in children with congenital heart disease (CHD) with its negative consequence has been recognized for a long time but in clinical practice the issue does not gain sufficient attention in developing countries.
\end{abstract}

Objectives: We conducted this study to investigate the prevalence of IDA in children with CHD and to assess the diagnostic values of hemogram, especially the erythrocyte indexes as a simple tool for early recognition of IDA.

Materials and methods: Children under 15 years old with confirmed CHD admitted to the Unit of Pediatric Cardiology, Cardiovascular Center, University Medical Center, Ho Chi Minh City from August 2016 to March 2017 were recr uited. The total blood cell count, serum iron, ferritin and transferrin were measured preoperatively.

Results: There were 69 pediatric patients were recruited in which 36 children with cyanotic CHD and 33 children with acyanotic CHD. There were $11.1 \%$ of children with cyanotic CHD and $3 \%$ of children with acyanotic CHD who showed true IDA. The prevalence of depletion or reduction of body iron store was very high. Up to $77.8 \%$ of children with cyanotic CHD and $87.2 \%$ children with acyanotic CHD were at risk of iron deficiency. The erythrocyte indexes proved to be useful as simple tool for detecting IDA in children with cyanotic CHD.

Conclusion: IN the present investigation, the prevalence of IDA was found high in the children with CHD. The total blood cell count and erythrocyte indexes were of suggestive value for diagnosis of IDA. Iron supplement treatment should be recommended in this group of patients.

Keywords: Iron deficiency anemia, Congenital heart disease, Children.

\section{Introduction}

Iron is an essential micronutrient playing a fundamental role in the formation of hemoglobin, myoglobin and numerous oxygen-carrier enzymes [1]. Iron deficiency anemia (IDA) is thought to be the most common micronutrient malnutrition in the world. According to the World Health Organization, IDA exerts negative impact on a great number of women and children in developing countries [2] and even in women of reproductive age in industrialized nations [1,3]. In 2005, there were 1.62 billion people, accounting for $24.8 \%$ of the global population, suffering anemia. Iron deficiency anemia was the most common cause and children of school age were the most vulnerable group [2]. Iron deficiency anemia reduces physical performance in adults and especially to exert negative impact on the psycho-motor development in children and adolescents [4,5]. Importantly, iron deficiency with or without anemia in infancy was associated with increased behavioral and social problems in adolescence [6]. Of note, IDA has been clearly shown to be an important risk factor that increases morbidity and mortality in patients with congenital heart disease (CHD) [7].
In children with $\mathrm{CHD}$, the right-to-left shunt leads to chronic arterial desaturation which in turn increases erythrocyte count and consequent high blood viscosity. In addition, in patients with cyanotic CHD suffering anemia, especially due to iron deficiency hypochromic anemia, red blood cells are less permeable. This phenomenon contributes in part to the increased rate of embolism and other cardiovascular accidents [8]. In children with cyanotic CHD, a hemoglobin ( $\mathrm{Hb})$ concentration in normal range might imply that patients are relatively anemic and this condition may have negative effects on mortality and morbidity $[9,10]$. An early detection and an appropriate treatment of anemia in patients with acyanotic heart disease who are presented with heart failure have been strongly recommended. In addition, the prevention of thromboembolic events and cyanotic spells in children with cyanotic CHD have been emphasized [11].

In industrialized countries, cyanotic CHD are currently operated in neonatal or infant period with optimal nutritional support. Whereas, in developing countries including Vietnam, an important number of children with complex CHD are not operated or completely operated. On top of it, the high 
prevalence of malnutrition is still a real public health concern [12]. We would speculate that IDA is of increased rate in this vulnerable group. Anemia in children with CHD, especially in cyanotic children who show high hematocrit value due to chronic hypoxia, is frequently missed or underdiagnosed in daily practice [13]. We conducted this investigation to assess the prevalence of IDA in children with CHD and to evaluate the usefulness of erythrocyte indexes in screening IDA in this group with the hope that these indexes would help physicians start the iron supplement more properly in remote health facilities where the specialized measurement of IDA is not routinely performed.

\section{Materials and Methods}

Children under 16 years old who were diagnosed with CHD and planned for elective cardiac surgery at Cardiovascular Center, University Medical Center, University of Medicine and Pharmacy at Ho Chi Minh City from August 2016 to March 2017 were recruited into the study. Children enrolled in this study were from different geographic areas in South Vietnam including Ho Chi Minh City, Mekong Delta, Central Highland and Central Coast Provinces. The study protocol was approved by the Scientific Board, University of Medicine and Pharmacy at Ho Chi Minh City. Diagnosis of CHD was confirmed by protocolized clinical and complementary examinations, especially by two independent echocardiograms performed by two pediatric cardiologists as well as cardiac multi-slice computerized tomography and cardiac catheterization when indicated. The final diagnosis reported in this study was from surgical report. We excluded patients who showed signs and symptoms of hemolysis due to thalassemia or other unknown mechanisms. We also excluded candidates who presented overt or suspected infection, fever, inflammatory diseases.

Blood cell count, serum iron, ferritin and transferrin were determined as the same time with preoperative tests on automatic analyzers at Department of Laboratory. Diagnosis of iron deficiency anemia was based on serum ferritin concentration. In this study, we used the value of $12 \mathrm{ng} / \mathrm{mL}$ as threshold for diagnosis of IDA. Patients with serum ferritin concentration under $50 \mathrm{ng} / \mathrm{mL}$ were considered to have iron storage depletion and value under $100 \mathrm{ng} / \mathrm{ml}$ as at risk of iron deficiency [14].
Data was imported to excel file and was analyzed with Graphpad Quickcalcs sofware. Results are presented in mean \pm standard deviation. The non-paired t-test was used for comparison between two group. The $2 \times 2$ table was used to calculate sensitivity and specificity. Value of $\mathrm{p}<0.05$ is considered statistically significant. To determine the optimal performance of an erythrocyte index as screening test, we used Youden's index to calculate the cut-off value.

\section{Results}

We recruited 69 children (37 males and 32 females) into the study. Thirty-six children were diagnosed to have cyanotic CHD and the rest 33 were of acyanotic CHD. Mean age was of $6.7 \pm 3.8$ years. Cyanotic group mean age was of $6.1 \pm 3.5$ and the acyanotic group $7.3 \pm 4.0$ years. The most common diseases in the cyanotic group (CG) was Tetralogy of Fallot (TOF) and in acyanotic group (AG) were ventricular septal defect (VSD) and atrial septal defect (ASD). There was no significant difference between two groups in terms of body weight and height. No patient had been given iron supplementation before admission. Table 1 presents blood cell counts and iron measurement results in two groups.

In the present study, we use serum ferritin as criteria to assess the body iron storage [14]. The stratification of body iron storage is presented in Table 2 .

There were only $22.2 \%$ children in cyanotic CHD group and $12.2 \%$ of those in acyanotic CHD group were not at risk of iron deficiency. Based on changes in erythrocyte indexes iron deficiency anemia is more easily recognized in children with acyanotic CHD as well as in children without cardiac disease. It is more challenging in children with cyanotic CHD. We compared erythrocyte indexes that may help to raise suspicion of iron deficiency in this group of patients. The analysis is shown in Table 3.

In order to assess the usefulness of erythrocyte indexes we tried to establish some cut off values based on some erythrocyte indexes that showed significant difference between two subgroups of cyanotic CHD children with and without iron depletion. Among the erythrocyte indexes examined, mean corpuscular hemoglobin $(\mathrm{MCH})$ and mean corpuscular hemoglobin concentration

Table 1: Blood cell counts and measurement of iron storage in two groups of patients. Numbers in brackets in second and third columns represent the min and max values of each parameter investigated. RBC: red blood cell count; $\boldsymbol{C D}$ : cyanotic group; AG: acyanotic group; MCV: Mean corpuscular volume; $\mathbf{M C H}$ : mean corpuscular hemoglobin; MCHC: mean corpuscular hemoglobin concentration, RDW: red blood cell distribution width.

\begin{tabular}{|c|c|c|c|}
\hline Parameters & $\begin{array}{l}\text { CG }(n=36) \\
(\text { Mix-Max) }\end{array}$ & $\begin{array}{l}\text { AG }(n=33) \\
\text { (Min-Max) }\end{array}$ & $P$ value \\
\hline SpO2 (\%) & $80.7 \pm 10.3(65-98)$ & $99.1 \pm 1.7(93-100)$ & 0.0001 \\
\hline $\mathbf{R B C}\left(\times 10^{12} / \mathrm{L}\right)$ & $6.4 \pm 1.2(4.4-9.7)$ & $4,8 \pm 0,5(4,0-6,2)$ & 0.0001 \\
\hline Hemoglobin (g/L) & $173.0 \pm 27.4(130-248)$ & $128,9 \pm 10,3(115-156)$ & 0.0001 \\
\hline Hematocrit (\%) & $52.2 \pm 7,9(39.4-68.0)$ & $39.1 \pm 3.2(34.5-47.6)$ & 0.0001 \\
\hline $\operatorname{MCV}(f L)$ & $82,8 \pm 7.2(63.1-93.2)$ & $81.3 \pm 6.7(58.7-94.0)$ & 0.3748 \\
\hline MCH (pg) & $27.6 \pm 3.5(19.5-40.4)$ & $26.8 \pm 2.4(18.5-31.1)$ & 0.2764 \\
\hline MCHC (\%) & $32.9 \pm 3.3(21.9-46.9)$ & $33.0 \pm 1.0(31.3-36.6)$ & 0.8678 \\
\hline RDW (\%) & $16.9 \pm 2.0(14.0-22.2)$ & $15.3 \pm 1.6(13.5-20.5)$ & 0.0005 \\
\hline White cell count $\left(\times 10^{9} / L\right)$ & $9.1 \pm 2.5(2.4-14.8)$ & $9.0 \pm 3.1(3.6-16.4)$ & 0.8805 \\
\hline Platelet count $\left(\times 10^{9} / L\right)$ & $253.8 \pm 64.6(105-369)$ & $284.1 \pm 74.2(114-412)$ & 0.0776 \\
\hline Serum iron $(\mu \mathrm{g} / \mathrm{dL})$ & $90.0 \pm 50.9(28-264)$ & $67.8 \pm 32.6(20-131)$ & 0.0390 \\
\hline Serum ferritin $(\mathrm{ng} / \mathrm{mL})$ & $70.5 \pm 52.5(5-233)$ & $76.7 \pm 59.1(5-351)$ & 0.6457 \\
\hline Serum transferrin $(\mathrm{g} / \mathrm{L})$ & $249.6 \pm 67.9(159-447)$ & $202.6 \pm 24.1(155.9-256)$ & 0.0003 \\
\hline
\end{tabular}


Table 2. Prevalence of different degrees of body iron storage and in two groups of patients.

\begin{tabular}{|c|c|c|c|}
\hline Serum ferritin $(\mathrm{ng} / \mathrm{mL})$ & Interpretation & $\begin{array}{c}\text { CG (36) (33) } \\
(n \text { \& percentage })\end{array}$ \\
\hline$<12$ & True IDA & $4(11.1 \%)$ \\
\hline $12-50$ & Depletion of body iron storage & $9(25.0 \%)$ & $7(3.0 \%)$ \\
\hline $50-100$ & At risk of iron deficiency & $15(41.7 \%)$ & $21.2 \%)$ \\
\hline$>100$ & Normal body iron storage & $8(22.2 \%)$ \\
\hline
\end{tabular}

Table 3. Erythrocyte indexes in children with cyanotic CHD with and without depletion of body iron storage (BIS) defined as serum ferritin $<50 \mathrm{ng} / \mathrm{mL}$. Comparison of erythrocyte indexes between two subgroups of children with cyanotic CHD: one subgroup with serum ferritin $<50 \mathrm{ng} / \mathrm{mL}$ and the other with serum ferritin $\geq 50 \mathrm{ng} / \mathrm{mL}$. $\boldsymbol{R B C}$ : red blood cell count; $\boldsymbol{S F}$ : serum ferritin; $\boldsymbol{M C V}$ : Mean corpuscular volume; $\boldsymbol{M C H}$ : mean corpuscular hemoglobin; MCHC: mean corpuscular hemoglobin concentration, $\boldsymbol{R D W}$ : red blood cell distribution width.

\begin{tabular}{|c|c|c|c|}
\hline Parameters & SF $<\mathbf{5 0} \mathbf{n g} / \mathbf{m L}$ & SF $\geq \mathbf{5 0} \mathbf{~ n g} / \mathbf{m L}$ & P value \\
\hline RBC $\left(\times 10^{12} / \mathrm{L}\right)$ & $7.1 \pm 1.4$ & $5.9 \pm 0.8$ & 0.0023 \\
\hline Hemoglobin $(\mathrm{g} / \mathrm{L})$ & $185.9 \pm 33.1$ & $166.6 \pm 21.3$ & 0.0403 \\
\hline Hematocrit $(\%)$ & $55.6 \pm 8.3$ & $50.3 \pm 7.2$ & 0.0526 \\
\hline MCV $(\mathrm{fL})$ & $79.1 \pm 9.3$ & $85.0 \pm 4.7$ & 0.0158 \\
\hline MCH $(\mathrm{pg})$ & $26.6 \pm 5.5$ & $28.2 \pm 1.5$ & 0.1944 \\
\hline MCHC $(\%)$ & $32.7 \pm 5.4$ & $33.1 \pm 1.1$ & 0.7312 \\
\hline RDW $(\%)$ & $17.9 \pm 2.5$ & $16.3 \pm 1.4$ & 0.0185 \\
\hline Serum iron $(\mu \mathrm{g} / \mathrm{dL})$ & $85.6 \pm 67.5$ & $94.0 \pm 40.1$ & 0.6408 \\
\hline Serum transferrin $(\mathrm{g} / \mathrm{L})$ & $292.1 \pm 91.2$ & $227.4 \pm 38.2$ & 0.0048 \\
\hline
\end{tabular}

(MCHC) are not significantly different between the two groups. Only erythrocyte indexes that reach the statistically significant difference are presented in Table 4.

\section{Discussion}

Iron deficiency and iron deficiency anemia are the most widespread micronutrient deficiency conditions in the world [1]. Iron deficiency anemia has been consistently shown to exert detrimental effects on physical performance in adults and psychomotor development in children [4]. In children with CHD who are already under negative restraints of multitude of pathological mechanisms that occur in association with the main disease, iron deficiency and IDA would have worse impact $[7,9,10]$. In developing countries, this situation is more pronounced with the prevalence of anemia among children under 4 years of age is estimated to range between $46 \%$ and $66 \%$ in developing countries and half of the cases is thought to be IDA [15]. The purposes of this study were to confirm again the importance of this condition and to encourage the prevention of IDA in community for this specific group of children.

In our investigation, $11.1 \%$ of children with cyanotic CHD demonstrated true IDA, even higher than acyanotic CHD group which showed $3 \%$ of true IDA $(p=0,1970)$. Up to $36.1 \%$ children in cyanotic group and $24.2 \%$ in acyanotic group were diagnosed with true IDA or showed depletion of body iron storage. Impressively, up to $77.8 \%$ children in cyanotic group and $87.8 \%$ in acyanotic group were at risk of iron deficiency. No patient in our study who had serum ferritin above $300 \mathrm{ng} / \mathrm{mL}$ which considered to be the upper normal range. These findings are truly worrisome. It is worth to notify that no patient was treated with iron supplement even though many of them had been repeatedly hospitalized in district or provincial hospitals.
Table 4. Ability of erythrocyte indexes in screening depleted iron storage in children with cyanotic CHD. Screening usefulness of some erythrocyte indexes in differentiating depleted body iron storage from non-depleted body iron storage in children with cyanotic CHD. RBC: red blood cell count; $\mathbf{M C V}$ : Mean corpuscular volume; $\mathbf{M C H}$ : mean corpuscular hemoglobin; MCHC: mean corpuscular hemoglobin concentration, DW: red blood cell distribution width; $\boldsymbol{S e}$ : sensitivity; Sp: specificity.

\begin{tabular}{|c|c|c|c|c|}
\hline Cut-off values & \multicolumn{2}{|c|}{ Se \& Sp } & $95 \% \mathrm{CD}$ & $\begin{array}{c}\text { Youden's } \\
\text { index }\end{array}$ \\
\hline \multirow{2}{*}{$\mathrm{RBC}=6 \times 10^{12} / \mathrm{L}$} & $\mathrm{Se}(\%)$ & 84.6 & $54.5-97.6$ & \multirow{2}{*}{0.3} \\
\hline & Sp (\%) & 47.8 & $26.8-69.4$ & \\
\hline \multirow{2}{*}{$\mathrm{Hb}=165 \mathrm{~g} / \mathrm{L}$} & $\mathrm{Se}(\%)$ & 76.9 & $46.2-94.7$ & \multirow{2}{*}{0.2} \\
\hline & $\mathrm{Sp}(\%)$ & 39.1 & $19.7-61.4$ & \\
\hline \multirow{2}{*}{ Hematocrit $=50 \%$} & Se $(\%)$ & 76.9 & $46.2-94.7$ & \multirow{2}{*}{0.2} \\
\hline & $\mathrm{Sp}(\%)$ & 43.5 & $23.2-65,5$ & \\
\hline \multirow{2}{*}{$\mathrm{MCV}=80 \mathrm{fL}$} & $\mathrm{Se}(\%)$ & 38.5 & $14.0-65.5$ & \multirow{2}{*}{0.2} \\
\hline & Sp (\%) & 82.6 & $61.2-94.9$ & \\
\hline \multirow{2}{*}{$\mathrm{RDW}=16 \%$} & Se $(\%)$ & 76.9 & $46.2-94.7$ & \multirow{2}{*}{0.2} \\
\hline & $\mathrm{Sp}(\%)$ & 47.8 & $26.8-69.4$ & \\
\hline
\end{tabular}

Given the fact that iron deficiency with or without anemia in infancy was associated with increased behavioral and social problems in adolescence [6], these findings would encourage the nutritional and iron supplement in this specular group of children with CHD who are at risk of higher problem with brain development [16].

In this study, we also notified that serum iron concentrations were lower and serum transferrin higher in patients with iron deficiency but these parameters showed a wide range of variation. It has been demonstrated that serum iron concentrations and serum transferrin are more prone to be affected by acute disease processes and/or infection than serum ferritin and serum ferritin should be the test of choice in diagnosis of IDA $[9,13]$.

In clinical practice one of evocating signs that raise the awareness of IDA is microcytic, hypochromic anemia. However, these changes are of limited value in screening IDA in children with cyanotic CHD due to polycythemia secondary to chronic hypoxemia. Some authors suggested that in this group of patients, hemoglobin concentration, hematocrit and red blood cell count are not useful indexes in diagnosis of IDA [17]. Contrarily, other believed that hemoglobin concentration $<150 \mathrm{~g} / \mathrm{L}$ and $\mathrm{MCV}<80 \mathrm{fL}$ may be considered anemic [13]. In the present study, children with cyanotic CHD showed clearly higher values of red blood cell counts, hemoglobin concentration, hematocrit compared to children with acyanotic CHD. This has been clearly confirmed in plenty of studies. However, the erythrocyte indexes such as $\mathrm{MCV}, \mathrm{MCH}$, and $\mathrm{MCHC}$ showed no significant difference between two groups.

In children with depletion of body iron storage, iron supplement must be started immediately to prevent true IDA. In the cyanotic group, comparison between the subgroup with depletion of body 
Citation: Binh TQ, Suong NTB, Dinh NH, et al. Iron deficiency anemia in children with congenital heart disease and the usefulness of erythrocyte indexes as a screening tool. Arch Gen Intern Med. 2018;2(3):1-4. DOI: 10.4066/ 2591-7951.1000049

iron storage (serum ferritin $<50 \mathrm{ng} / \mathrm{mL}$ ) and the subgroup with serum ferritin $<50 \mathrm{ng} / \mathrm{mL}$ clearly showed that red blood cell counts, hemoglobin concentration, hematocrit, and red blood cell distribution width were higher in iron-depleted subgroup. In parallel, mean corpuscular volume was lower in irondepleted subgroup. This implies that the iron-depleted subgroup demonstrated a polycythemia, wide range of erythrocyte sizes. Differently stated, erythropoietic process in the iron-depleted subgroup was less efficient in comparison to that in patients whose iron storage was not depleted. This finding, once again, emphasizes the important role of prompt iron and nutritional supplement. We have tried to look for erythrocyte indexes that help the clinical suspicion of iron deficiency in children with cyanotic CHD. However, these indexes were of limited sensitivity and specificity although cut-off values of RBC, Hb, Hct, RDW of $6 \times 1012 / \mathrm{L}, 165 \mathrm{~g} / \mathrm{L}, 50 \%, 16 \%$ respectively were of acceptable sensitivity and MCV of $80 \mathrm{fL}$ was of good specificity. We would not be able to comment on these values of erythrocyte indexes for the lack of conclusive distinguishing power.

Nevertheless, when looking at results of serum ferritin in this study, we could easily notice that a majority of children were of IDA, iron depletion or at risk of iron deficiency while only small percentage of children having iron storage in normal range and no patient ware at risk of iron overload. Hence, a serious consideration of a nutrient and iron supplement to all children with CHD might seem appropriate.

\section{Conclusion}

Iron deficiency and iron deficiency anemia are commonly encountered in children with congenital heart disease. Both groups of cyanotic and acyanotic CHD are equally at high risk of IDA and depletion of body iron storage. The more severe the iron deficiency is, the less efficient the erythropoietic process which manifests as polycythemia, increased hemoglobin concentration, hematocrit, and red blood cell distribution width. Detection and prevention of iron deficiency and IDA are not sufficiently emphasized and practiced in South Vietnam. Careful analysis of hemogram may help to increase the rate of early detection and prevention or treatment of iron deficiency or IDA in children with CHD in order to alleviate cardiac mortality and morbidity as well as to foster psychomotor development. In remote regions where specialized measure of body iron storage is still not available, an empiric iron supplement should be considered in presence of other clinical manifestations of IDA before a referral to specialized centers should be considered. Large-scale studies investigating the prevalence of IDA and the effectiveness of iron supplement in this group of patients are warranted.

\section{References}

1. Morrone A, Nosotti L, Piombo L, et al. Iron deficiency anaemia prevalence in a population of immigrated women in Italy. Eur J Public Health. 2010;22:256-62.

2. de Benoist B, McLean E, Egli I, et al. Worldwide prevalence of anaemia 1993-2005: WHO global database on anaemia. World Health Organization 2008. ISBN 9789241596657.

3. Ahmed F, Coyne T, Dobson A, et al. Iron status among
Australian adults: findings of a population based study in Queensland, Australia. Asia Pac J Clin Nutr. 2008;17:40-7.

4. Killip S, Bennett JM, Chambers MD. Iron deficiency anemia. Am Fam Physician. 2007;75:671-8.

5. Lozoff B. Iron deficiency and child development. Food Nutr Bull. 2007;28(4 Suppl):S560-71.

6. Doom JR, Richards B, Caballero G, et al. Infant Iron Deficiency and Iron Supplementation Predict Adolescent Internalizing, Externalizing, and Social Problems. J Pediatr. 2018;195:199-205.

7. Carson JA, Duff A, Poses RM, et al. Effect of anemia and cardiovascular disease on surgical mortality and morbidity. Lancet. 1996;348:1055-60.

8. Broberg CS, Bax BE, Okonko DO, et al. Blood viscosity and its relationship to iron defciency, symptoms and exercise capacity in adults with cyanotic congenital heart disease. J Am Coll Cardiol. 2006;48:356-65.

9. Olcay L, Ozer S, Gurgey A, et al. Parameters of iron deficiency in children with cyanotic congenital heart disease. Pediatr Cardiol. 1996;17:150-4.

10. Roodpeyma S, Movssavi F, Kamali Z. Red blood cell indices in cyanotic congenital heart disease. Med J Iran Hosp. 2002;4:24-6.

11. Paul S, Paul RV. Anemia in heart failure, implications, management and outcomes. J Cardiovasc Nurs. 2004;19:S57-66.

12. Kramer CV, Allen S. Malnutrition in developing countries. Paediatrics and Child Health. 2015;25:422-7.

13. Amoozgar H, Soltani M, Besharati A, et al. Undiagnosed Anemia in Pediatric Patients with Congenital Heart Diseases. Iran Cardiovasc Res J. 201;5:69-70.

14. Parischa S-RS, Flecknoe-Brown SC, Allen KJ, et al. Diagnosis and management of iron deficiency anemia: a clinical update. MJA. 2010;193:525-32.

15. Stoltzfus RJ, Mullany L, Black RE. Iron deficiency anaemia. In: Ezzati M, Lopez AD, Rodgers A, Murray CJL, eds. Comparative quantification of health risks: Global and regional burden of disease attribution to selected major risk factors. Vol 1. Geneva: World Health Organization. 2004:163-209.

16. McQuillen PS, Miller SP. Congenital heart disease and brain development. Ann N Y Acad Sci. 2010;1184:68-86.

17. Onur CB, Sipahi T, Tavil B, et al. Diagnosing iron deficiency in cyanotic heart disease. Indian J Pediatr. 2003;70:29-31.

\section{*Correspondence to:}

Le Minh Khoi

Cardiovascular Center

University Medical Center

University of Medicine and Pharmacy at Ho Chi

Minh City

Vietnam

E-mail: leminhkhoimd@gmail.com 